\title{
The quantum optical description of three experiments involving non-linear optics using a graphical method
}

\author{
Stefan Ataman ${ }^{\mathrm{a}}$ \\ e-mail: ataman@ece.fr \\ Received: date / Revised version: date

\begin{abstract}
In this paper we describe and thoroughly discuss three reported experiments in quantum optics (QO) involving interferometers and non-linear crystals. We show that by using a graphical method and an over-simplified model of the parametric down-conversion process, we arrive to explain all the important results reported in the respective papers. Indistinguishability is discussed in the case of separable/nonseparable (i.e. entangled) quantum systems and our interpretation is sometimes at variance with the one given by the authors reporting the experiments.
\end{abstract}

PACS. PACS-key describing text of that key - PACS-key describing text of that key

\section{Introduction}

Indistinguishability in Quantum Mechanics plays a key role in many experiments. Indeed, adding quantum amplitudes associated with the indistinguishable paths rather than probabilities, allows one to explain the observed interference phenomena $[1,2,3,4]$.

The fact that in a Mach-Zehnder interferometer single quanta of light are in a coherent superposition of being simultaneously in both arms has many counter-intuitive consequences, among others the "click by click" buildup of the interference pattern [5] or the the so-called "interactionfree measurements" $[6,7]$.

The non-linear process of spontaneous parametric downconversion (SPDC) [8] provides pairs of highly entangled photons $[9,10,11,12]$. It had been used hitherto in many experiments in quantum optics $[1,2,3,13,14,15,16,17,18$, 19]. One could emphasize the fundamental experiments demonstrating the non-locality of quantum mechanics $[13$, $14]$.

In an experiment by $\mathrm{Ou}$, Wang, Zou and Mandel (proposed in [15] and reported in [16]), two non-linear crystals fed by the same pump laser through a beam splitter perform down-conversions. By slightly varying the position of the beam splitter (and therefore the relative phase of the pump field between the crystals), an interference pattern is recovered. The authors model this by considering a coherent superposition between the output wavepacket and the vacuum state. We will arrive at the same results using a simpler model, not needing to resort to this "phase memory" of the vacuum.

Another experiment using two non-linear crystals showing a quite counter-intuitive behavior was reported by

\footnotetext{
a Alternative e-mail address: stefan.ataman@gmail.com
}

Zou, Wang and Mandel in [17] and its theoretical model further refined in [18]. This time, although the idler beams of the two non-linear crystals are discarded, they seem to play a major role in the interference of the other beams. The apparent paradox of this experiment will be analyzed later on.

Based on the principle of the previous experiment, the rather surprising idea of imaging an object with undetected photons was recently reported in an experiment [19], where a series of small objects has been imaged using undetected (discarded) photons and arrays of photon counters that detected photons not crossing the object's path.

In this paper we describe three experiments involving non-linear crystals using the graphical method introduced in [20]. Although a very simple model for the non-linear process of SPDC is used, all important phenomena reported in the respective papers can be accounted for. The experiments are thoroughly discussed, showing were an explanation differing from the authors' one can be given.

This paper is organized as follows. In Section 2 we give a theoretical justification for the field operator transformations and supplement the graphical method introduced in [20] with the inclusion of the non-linear quantum optical phenomenon of spontaneous parametric down-conversion. The experiment of Ou, Wang, Zou and Mandel [16] is described using the graphical method in Section 3 and the results compared to the ones experimentally obtained. The more counter-intuitive experiment of Zou, Wang and Mandel [17] is discussed in Section 4. Using a similar experimental setup, the imaging of an object with undetected photons [19] is described and discussed in Section 5. Finally, conclusions are drawn in Section 6. 


\section{Field operator transformations in quantum optics}

Throughout this paper, we shall consider an input state derived from the pump laser. Since we select only events where a down-conversion takes place, we can write the input state as

$$
\left|\psi_{i n}\right\rangle=\left|1_{p}\right\rangle=\hat{a}_{p}^{\dagger}|0\rangle
$$

where $\left|1_{p}\right\rangle$ denotes a Fock state with one photon in mode (port) $p, \hat{a}_{p}^{\dagger}$ denotes the input field creation operator and $|0\rangle$ is the vacuum state. In order to find the output state, we need an operator transformation function $g$ connecting the input creation field operator to the output ones,

$$
\hat{a}_{p}^{\dagger}=g\left(\hat{a}_{s}^{\dagger}, \hat{a}_{s^{\prime}}^{\dagger}, \hat{a}_{i}^{\dagger}, \hat{a}_{i^{\prime}}^{\dagger}\right)
$$

where $\hat{a}_{k}^{\dagger}$ is the creation operator for the output port $k$ with, $k=\left\{s, s^{\prime}, i, i^{\prime}\right\}$. Therefore, at least formally, the output state can be written as

$$
\left|\psi_{\text {out }}\right\rangle=g\left(\hat{a}_{s}^{\dagger}, \hat{a}_{s^{\prime}}^{\dagger}, \hat{a}_{i}^{\dagger}, \hat{a}_{i^{\prime}}^{\dagger}\right)|0\rangle
$$

In [20] a graphical method allowing the fast computation of field operator transformation has been introduced for linear optical systems. We extend this method to nonlinear transformations by including the SPDC process.

The SPDC process produces two entangled single photon wave packets $[9,11]$ that are generally very short[1] (hence non-monochromatic). The input beam, generally called the pump $(p)$ is split into two output beams, called the signal $(s)$ and the idler $(i)$. Energy $(\hbar \omega)$ and momentum $(\hbar \boldsymbol{k})$ conservation relations impose $\omega_{p}=\omega_{s}+\omega_{i}$ and $\boldsymbol{k}_{p} \approx \boldsymbol{k}_{s}+\boldsymbol{k}_{i}$ (the latter being also called "phase matching" condition). In this paper, a very simplified model of the SPDC process will be employed, where this process converts a monochromatic input photon from the pump field into two (equally monochromatic) photons in the signal, and, respectively, idler outputs. From the perspective of $\mathrm{QO}$, the field operator transformations in the SPDC process are

$$
\hat{a}_{p}^{\dagger}=\gamma \hat{a}_{s}^{\dagger} \hat{a}_{i}^{\dagger}
$$

where $\gamma$ is a parameter connected to the $\chi^{(2)}$ non-linearity of the optical medium. For simplicity, throughout this paper we will consider ${ }^{1} \gamma=1$, hence equation (4) transforms the input state (1) into

$$
\left|\psi_{\text {out }}\right\rangle=\hat{a}_{s}^{\dagger} \hat{a}_{i}^{\dagger}|0\rangle=\left|1_{s} 1_{i}\right\rangle
$$

at the output of the non-linear crystal.

\footnotetext{
${ }^{1}$ In practice we have $\gamma \sim 10^{-6}$. However, the choice of $\gamma=1$ in our analysis can be justified by the fact that we only consider events where a down-conversion actually takes place.
}

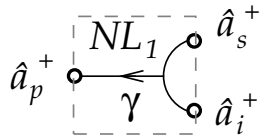

Fig. 1. The graphical description of the non-linear process of spontaneous parametric down-conversion. The input (creation) field operator $\hat{a}_{p}^{\dagger}$ can be written as a product of the two output (creation) field operators, $\hat{a}_{s}^{\dagger}$ and $\hat{a}_{i}^{\dagger}$.

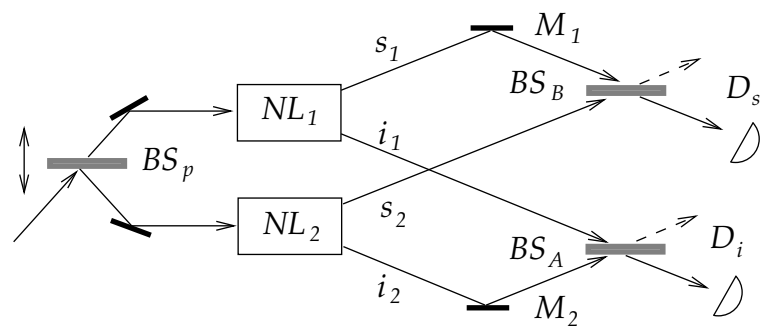

Fig. 2. The Ou, Wang, Zou and Mandel experiment [16]. The interfering paths $s_{1}-s_{2}$ and $i_{1}-i_{2}$ are made as equal as possible. The beam splitter $\mathrm{BS}_{p}$ is slightly moved as indicated by the arrow causing a phase difference between the two pump fields.

\section{The experiment of Ou, Wang, Zou and Mandel}

The experiment performed by Ou, Wang, Zou and Mandel [16] is depicted in Fig. 2. A pump laser beam is divided by the beam splitter $\mathrm{BS}_{p}$ between two non-linear crystals, denoted $N L_{1}$ and $N L_{2}$. The signal and, respectively, idler beams from the two crystals are brought together at beam splitters $\mathrm{BS}_{B}$ and, respectively, $\mathrm{BS}_{A}$. The optical path lengths from the crystals to the beam splitters were made as equal as possible. The mixed signal $\left(s_{1}\right.$ and $\left.s_{2}\right)$ and, respectively, idler $\left(i_{1}\right.$ and $\left.i_{2}\right)$ photons are then sent to the photo-detectors $D_{s}$, and, respectively, $D_{i}$. The two-photon coincident detection rates were measured in respect with the displacement of $\mathrm{BS}_{p}$ (Fig. 3 from [16]) and a sinusoidal variation was found.

In Fig. 3 the same experiment in described using the graphical method [20]. The three beam splitters $\mathrm{BS}_{p}, \mathrm{BS}_{A}$ and $\mathrm{BS}_{B}$ (assumed identical), are depicted by the three "butterflies". The beam splitters are assumed to have a transmission (reflection) coefficient $T(R)$. Energy conservation imposes the well-known constraints $|T|^{2}+|R|^{2}=1$ and $T^{*} R+T R^{*}=0[21]$. The variable path length between $\mathrm{BS}_{p}$ and the two non-linear crystals is modelled by the phase shift ${ }^{2} \mathrm{e}^{i \varphi}$. The non-linear crystals are depicted using the graph introduced in Fig. 1.

In order to express $\hat{a}_{p}^{\dagger}$ in respect with the output field operators we examine the paths connecting them in Fig. 3. From the crystal $N L_{1}$ to $\hat{a}_{p}^{\dagger}$ we have a factor $T \mathrm{e}^{i \varphi}$. The signal (upper) port of $N L_{1}$ is connected to $\hat{a}_{s}^{\dagger}$ with a coef-

2 We wish to express the input creation operator in respect with the output field operators, therefore all arrows point "backwards in time". This is why the phase shift is $\mathrm{e}^{i \varphi}$ and not $\mathrm{e}^{-i \varphi}$. 


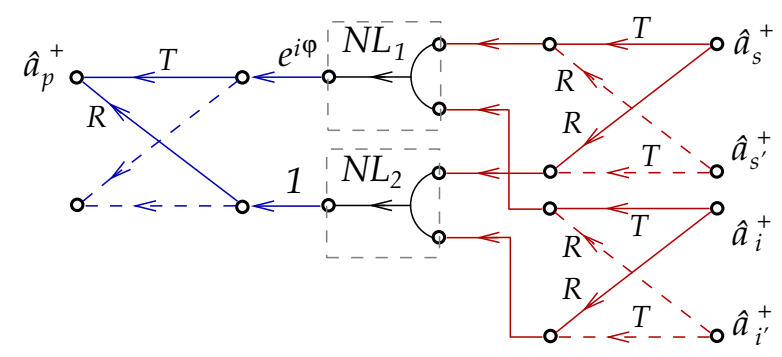

Fig. 3. (Color online) The graphical description of the $\mathrm{Ou}$, Wang, Zou and Mandel experiment. The beam splitters are represented as "butterflies". Ports connected to dashed paths are either unused or will be traced out in the density matrix operator. The $\mathrm{e}^{i \varphi}$ coefficient models the path length difference between $\mathrm{BS}_{p}-N L_{1}$ and $\mathrm{BS}_{p}-N L_{2}$.

ficient $T$ and to $\hat{a}_{s^{\prime}}^{\dagger}$ with a coefficient $R$. The idler (lower) port of $N L_{1}$ is connected to $\hat{a}_{i}^{\dagger}$ via a coefficient $T$ and to $\hat{a}_{i^{\prime}}^{\dagger}$ via a coefficient $R$. Putting together these contributions, one ends up with $T \mathrm{e}^{i \varphi} \times\left(T \hat{a}_{s}^{\dagger}+R \hat{a}_{s^{\prime}}^{\dagger}\right) \times\left(T \hat{a}_{i}^{\dagger}+R \hat{a}_{i^{\prime}}^{\dagger}\right)$. Similar arguments allow us to add the contribution of the second crystal and we obtain the field operator transformation

$$
\begin{array}{r}
\hat{a}_{p}^{\dagger}=T \mathrm{e}^{i \varphi}\left(T \hat{a}_{s}^{\dagger}+R \hat{a}_{s^{\prime}}^{\dagger}\right)\left(T \hat{a}_{i}^{\dagger}+R \hat{a}_{i^{\prime}}^{\dagger}\right) \\
+R\left(R \hat{a}_{s}^{\dagger}+T \hat{a}_{s^{\prime}}^{\dagger}\right)\left(R \hat{a}_{i}^{\dagger}+T \hat{a}_{i^{\prime}}^{\dagger}\right)
\end{array}
$$

or, in other words, we got the function $g$ from equation (2). All we have to do now is apply this result to equation (3). After some simplifications and assuming all beam splitters to be balanced ( $T=1 / \sqrt{2}$ and $R=i / \sqrt{2}$ [21]), we get

$$
\begin{aligned}
\left|\psi_{\text {out }}\right\rangle= & \frac{1}{\sqrt{2}}\left(\sin \left(\varphi^{\prime}\right)\left|1_{s} 1_{i}\right\rangle+\cos \left(\varphi^{\prime}\right)\left|1_{s^{\prime}} 1_{i}\right\rangle\right. \\
& \left.+\cos \left(\varphi^{\prime}\right)\left|1_{s} 1_{i^{\prime}}\right\rangle-\sin \left(\varphi^{\prime}\right)\left|1_{s^{\prime}} 1_{i^{\prime}}\right\rangle\right)
\end{aligned}
$$

where we ignored a common phase factor and denoted $\varphi^{\prime}=\varphi / 2-\pi / 4$.

We could now simply project the output state vector given by equation (7) into the $\left|1_{s} 1_{i}\right\rangle$ state and obtain the probability of coincident counts $P_{s-i}$. However, for the sake of generality, we shall employ the density matrix approach, outlined in Appendix A. Therefore, we define the output density matrix operator $\hat{\rho}_{\text {out }}=\left|\psi_{\text {out }}\right\rangle\left\langle\psi_{\text {out }}\right|$ and trace it over the two unused outputs $\left(s^{\prime}\right.$ and $\left.i^{\prime}\right)$ ending up with the partial trace operator $\hat{\rho}_{s, i}=\operatorname{Tr}_{s^{\prime}, i^{\prime}}\left\{\hat{\rho}_{\text {out }}\right\}$ given in equation (22). The probability of coincident counts ${ }^{3}$ at the detectors $D_{s}$ and $D_{i}$ is now easily computed as

$$
P_{s-i}=\operatorname{Tr}\left\{\hat{a}_{s}^{\dagger} \hat{a}_{s} \hat{a}_{i}^{\dagger} \hat{a}_{i} \hat{\rho}_{s, i}\right\}=\frac{1}{4}(1+\sin (\varphi))
$$

and we find indeed an interference pattern while varying the position of the beam splitter $\mathrm{BS}_{p}$. If we replace the phase $\varphi$ by $k_{p} z_{p}$ where $k_{p}$ is the wavenumber of the

\footnotetext{
3 Throughout this paper we assume ideal photo-detectors.
}

pump laser and $z_{p}$ is the path length difference, we end up with the interference pattern $P_{s-i} \sim 1+\cos \left(k_{p} z_{p}\right)$ i.e. the interference fringes are periodic with the pump field frequency. This is consistent with what is reported in the paper (Fig. 3 in [16]).

The authors conclude "[...] we have demonstrated that the light produced in the down-conversion process carries information about the pump phase through entanglement with the vacuum." [16]. While this is one way to explain the experiment, it might also be discussed using the simple model used throughout this section. Fock states have indeed ill-defined phases, but we can give an operational meaning to a phase difference between the quantum superposition of two Fock states.

It is interesting to note that for the probability of single counts at, say, detector $D_{s}$ one obtains

$$
P_{s}=\operatorname{Tr}\left\{\hat{a}_{s}^{\dagger} \hat{a}_{s} \hat{\rho}_{s}\right\}=\frac{\sin ^{2}\left(\varphi^{\prime}\right)+\cos ^{2}\left(\varphi^{\prime}\right)}{2}=\frac{1}{2}
$$

where $\hat{\rho}_{s}=\operatorname{Tr}_{i}\left\{\hat{\rho}_{s, i}\right\}$ and no interference fringes can be found on varying $\varphi$. This is about to change in the next section, where a modified version of this experiment will be discussed.

\section{The experiment of Zou, Wang and Mandel}

In [17], Zou, Wang and Mandel performed an experiment that has been dubbed "mind boggling" [22]. The counterintuitive part in this experiment comes from the fact that some photons interfere or not conditioned on the distinguishability of other, undetected photons.

The experiment is depicted in Fig. 4. Similar to the previous experiment, a pump laser is divided by a beam splitter between two non-linear crystals. The two signal beams $\left(s_{1}\right.$ and $\left.s_{2}\right)$ are brought together in the beam splitter $\mathrm{BS}_{o}$ and a photo-detector $D_{s}$ is placed at its output. The idler beam of the first crystal $\left(i_{1}\right)$ is passed through the second one (the crystals are transparent, therefore attenuation is negligible) and superposed on the idler beam $i_{2}$. A detector $D_{i}$ is placed at the output idler beam. Given the configuration of this experiment, any detection at $D_{i}$ is unable to pinpoint the origin of the light quantum. What Zou, Wang and Mandel observed was an interference pattern on monitoring the singles detection rate at $D_{s}$, while varying the path length difference by moving the beam splitter $\mathrm{BS}_{o}$. Up to this point, no "mind boggling" features showed up in this experiment: the singles rate is the result of an interference between the undistinguishable paths of an interferometer composed of $\mathrm{BS}_{p}, N L_{1}, N L_{2}$ and $\mathrm{BS}_{o}$.

However, if we block the beam $i_{1}$ (or simply make it distinguishable in respect with $i_{2}$ ), the interference pattern disappears. This result is rather surprising since the idler beams do not participate to the interference happening at the beam splitter $\mathrm{BS}_{o}$.

In Fig. 5 we describe this experiment using the graphical method. As before, the two beam splitters (assumed identical) are depicted by the two "butterflies" while the path length difference in the interferometer formed by the 


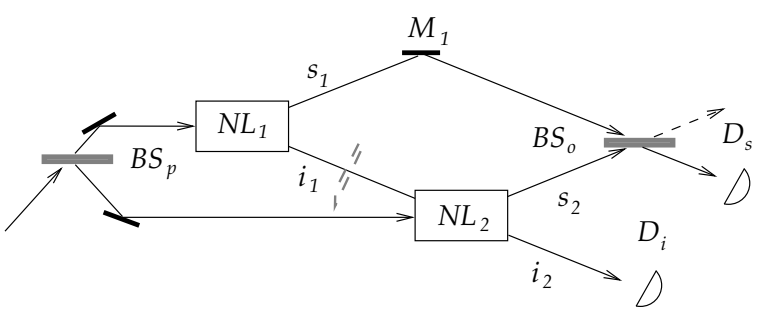

Fig. 4. The Zou, Wang and Mandel experiment [17]. The idler beam from the non-linear crystal $N L_{1}$ is aligned so that it will overlap with the idler beam from $N L_{2}$. Thus, indistinguishability of the idler beams is assured at the detector $D_{i}$.

two non linear crystals and the beam splitter $\mathrm{BS}_{o}$ is modelled by the phase shift $\mathrm{e}^{i \varphi}$. By a simple inspection of the graph we can express contribution to the the field operation transformation of the upper path (the one containing $\left.N L_{1}\right)$ as $T \times\left(T \hat{a}_{s}^{\dagger}+R \hat{a}_{s^{\prime}}^{\dagger}\right) \times \hat{a}_{i}^{\dagger}$. Adding the contribution of the second crystal yields the final result

$$
\hat{a}_{p}^{\dagger}=\left(T^{2}+\mathrm{e}^{i \varphi} R^{2}\right) \hat{a}_{s}^{\dagger} \hat{a}_{i}^{\dagger}+T R\left(1+\mathrm{e}^{i \varphi}\right) \hat{a}_{s^{\prime}}^{\dagger} \hat{a}_{i}^{\dagger}
$$

and for the case of balanced beam splitters $(T=1 / \sqrt{2}$ and $R=i / \sqrt{2}$ ) we end up with the output state

$$
\left|\psi_{\text {out }}\right\rangle=-\sin (\varphi / 2)\left|1_{s} 1_{i}\right\rangle+\cos (\varphi / 2)\left|1_{s^{\prime}} 1_{i}\right\rangle
$$

where we ignored a common phase factor. We compute again the density matrix $\hat{\rho}_{\text {out }}=\left|\psi_{\text {out }}\right\rangle\left\langle\psi_{\text {out }}\right|$ and partially trace it over the unused output $\left(s^{\prime}\right)$ yielding

$\hat{\rho}_{s, i}=\sin ^{2}(\varphi / 2)\left|1_{s} 1_{i}\right\rangle\left\langle 1_{s} 1_{i}\left|+\cos ^{2}(\varphi / 2)\right| 0_{s} 1_{i}\right\rangle\left\langle 0_{s} 1_{i}\right|$

The probability of coincident counts at the detectors $D_{s}$ and $D_{i}$ is immediately obtained as

$$
P_{s-i}=\operatorname{Tr}\left\{\hat{a}_{s}^{\dagger} \hat{a}_{s} \hat{a}_{i}^{\dagger} \hat{a}_{i} \hat{\rho}_{s, i}\right\}=\sin ^{2}(\varphi / 2)
$$

and not surprisingly one finds an interference pattern. If we write the phase shift $\varphi$ as $k_{s} z_{s}$, where $k_{s}$ is the wavenumber in the $s$ mode and $z_{s}$ denotes the path length difference of the signal beams to the beam splitter, the coincident rate becomes $P_{s-i} \sim 1 / 2\left(1-\cos \left(k_{s} z_{s}\right)\right)$, consistent with the observed frequency of the interference fringes (Fig. 2 in [18]).

However, the counter-intuitive feature of this experiment does not come from the coincident counts. Indeed, by computing the singles detection rate at detector $D_{s}$ one gets

$$
P_{s}=\operatorname{Tr}\left\{\hat{a}_{s}^{\dagger} \hat{a}_{s} \hat{\rho}_{s}\right\}=\sin ^{2}(\varphi / 2)
$$

where $\hat{\rho}_{s}=\operatorname{Tr}_{i}\left\{\hat{\rho}_{s, i}\right\}$ and this time, contrary to equation (9), there is an interference pattern present on varying $\varphi$.

A natural question arises: why in this experiment the singles detection rate at $D_{s}$ yielded an interference pattern while in equation (9) it did not? We could argue that in both cases, a detection at $D_{s}$ or $D_{i}$ resulted from two indistinguishable paths, therefore if we apply the dictum

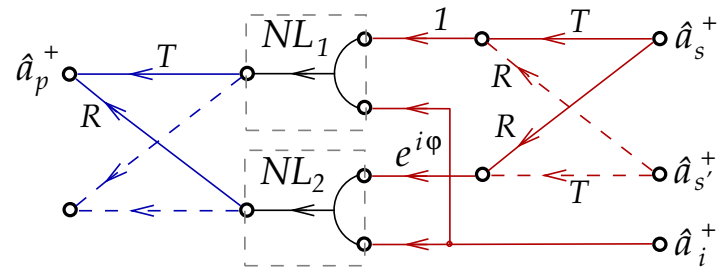

Fig. 5. (Color online) The graphical description of the Zou, Wang and Mandel experiment [17]. Ports connected to dashed paths are either unused or will be traced out in the density matrix operator. The two idler beams have been connected together and they end up at the port $\hat{a}_{i}^{\dagger}$.

"ignorance is interference" this result should be rather surprising.

A logical guess would be that the field from $i_{1}$ induced down-conversions in the crystal $N L_{2}$. As the authors pointed out [17], this is tenable if the field intensities are high enough. However, the same phenomenon is present for very low intensities [23]. It is found that the down-conversions in $N L_{1}$ and $N L_{2}$ are spontaneous (thus uncorrelated) even when an idler photon from $i_{1}$ crosses $N L_{2}$. This conclusion is strengthened in [19], where the authors experimentally showed that induced emission in $N L_{2}$ plays no role.

The authors conclude in their paper that "in quantum mechanics interference is always a manifestation of the intrinsic indistinguishability of the photon paths, in which case the corresponding probability amplitudes add. [...] once the $i_{1}, i_{2}$ connection is broken it becomes feasible, in principle, to determine from the counts [...] $D_{i}$ whether the detected signal photon comes from $N L_{1}$ or $N L_{2}$, and this destroys the interference" [17]. While this affirmation is certainly not wrong, it cannot contain the whole story; if it would, equation (9) should have shown an interference pattern, too.

It is well known that a composite system whose global wavevector can be written in a factorized form allows the separate analysis of each subsystem. This is not the case for an entangled system, where each subsystem cannot be discussed separately. Therefore, besides indistinguishability, one has to consider if each subsystem (the signal and idler beams in this case) can be analyzed separately ${ }^{4}$ or not. In the case of equation (11) the answer is affirmative, yielding the factorized form

$$
\left|\psi_{\text {out }}\right\rangle=\left(-\sin \left(\frac{\varphi}{2}\right)\left|1_{s}\right\rangle+\cos \left(\frac{\varphi}{2}\right)\left|1_{s^{\prime}}\right\rangle\right) \otimes\left|1_{i}\right\rangle
$$

hence the interference pattern obtained in the singles detection rate $P_{s}$ in equation (14). This factorization is impossible in the case of equation (7), therefore in this case the subsystem of the signal beams only cannot be treated separately, as proven by the missing interference pattern in equation (9).

4 A more involved discussion about non-separable systems and the effect of a partial measurement is done in reference [24]. 


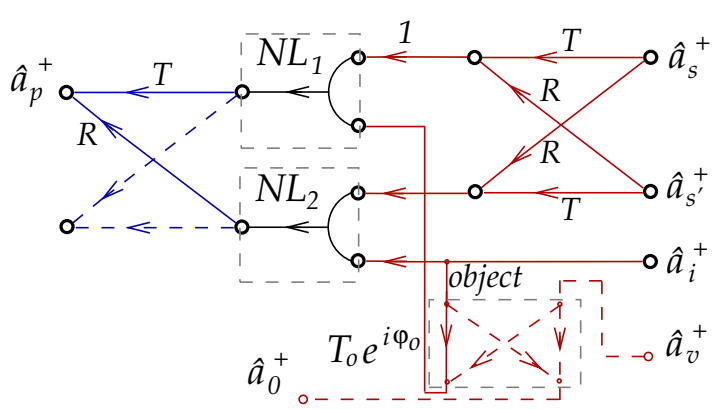

Fig. 6. (Color online) The graphical description of the Lemos et al. experiment [19]. Attenuation is QO is modelled through a beam splitter. The two idler beams are connected through the attenuation loss $T_{o}$ and phase shift $\mathrm{e}^{i \varphi_{o}}$ introduced by the object to be imaged. However, the empty input mode $\hat{a}_{0}^{\dagger}$ as well as the discarded output mode $\hat{a}_{v}^{\dagger}$ have to be taken into account.

\section{Imaging an object with undetected photons}

In a recent experiment [19], the same principle used by Zou, Wang and Mandel was implemented in order to image an object with undetected photons. The experiment (Figure 1 in [19]) is almost identical in principle to the one depicted in Fig. 4 and the object to be imaged is placed in the dashed region on the beam $i_{1}$. Therefore, depending on how the beam $i_{1}$ is attenuated and/or dephased before entering the crystal $N L_{2}$, the indistinguishability of the two idler beans will me more or less perfect.

In Fig. 6 we give the graphical representation of this experiment. The idler beams are now connected through a factor $T_{o} \mathrm{e}^{i \varphi}$ where $T_{o}$ and, respectively, $\mathrm{e}^{i \varphi_{o}}$, model the transmittance and, respectively, the phase shift introduced by the object intended to be imaged. For a perfectly transparent/opacque object we have $T_{o}=1 / 0$. In order to have a unitary evolution, the empty input mode $\hat{a}_{0}^{\dagger}$ as well as the "absorbed" output mode $\hat{a}_{v}^{\dagger}$ have to be taken into account. Inspecting the graph from Fig. 6 allows one to write down the field operator transformation yielding

$$
\begin{aligned}
\hat{a}_{p}^{\dagger}=T\left(T \hat{a}_{s}^{\dagger}+R \hat{a}_{s^{\prime}}^{\dagger}\right) & \mathrm{e}^{i \varphi_{o}}\left(T_{o} \hat{a}_{i}^{\dagger}+R R_{o} \hat{a}_{v}^{\dagger}\right) \\
& +R\left(R \hat{a}_{s}^{\dagger}+T \hat{a}_{s^{\prime}}^{\dagger}\right) \hat{a}_{i}^{\dagger}
\end{aligned}
$$

and assuming again balanced beam splitters we obtain the output state vector

$$
\begin{array}{r}
\left|\psi_{\text {out }}\right\rangle=\frac{T_{o} \mathrm{e}^{i \varphi_{o}}-1}{2}\left|1_{s} 1_{i}\right\rangle+\frac{R_{o} \mathrm{e}^{i \varphi_{o}}}{2}\left|1_{s} 1_{v}\right\rangle \\
+i \frac{T_{o} \mathrm{e}^{i \varphi_{o}}+1}{2}\left|1_{s^{\prime}} 1_{i}\right\rangle+i \frac{R_{o} \mathrm{e}^{i \varphi_{o}}}{2}\left|1_{s^{\prime}} 1_{v}\right\rangle
\end{array}
$$

Writing the output density matrix $\hat{\rho}_{\text {out }}=\left|\psi_{\text {out }}\right\rangle\left\langle\psi_{\text {out }}\right|$ and tracing out the unused output ports $\left(s^{\prime}, i\right.$ and $\left.v\right)$ gets us to $\hat{\rho}_{s}=\operatorname{Tr}_{s^{\prime}, i, v}\left\{\hat{\rho}_{\text {out }}\right\}$. The probability of singles detection at $D_{s}$ is given by

$$
P_{s}=\operatorname{Tr}\left\{\hat{a}_{s}^{\dagger} \hat{a}_{s} \hat{\rho}_{s}\right\}=\frac{1-T_{o} \cos \left(\varphi_{o}\right)}{2}
$$

where we used the fact that $\left|T_{o}\right|^{2}+\left|R_{o}\right|^{2}=1$ and assumed $T_{o}$ real. Therefore, both the transmissivity and the phase shift introduced by the object modify the singles detection rate at $D_{s}$. For example, for a transparent object we get

$$
P_{s}=\frac{1}{2}\left(1-\cos \left(\varphi_{o}\right)\right)
$$

and a phase shift of $\pi$ radians can be clearly imaged, while a $2 \pi$ phase shift cannot. This is consistent with what was experimentally found (Fig. 5 in [19]).

\section{Conclusions}

In this paper we employed a graphical method and a simple model of the spontaneous parametric down-conversion in order to describe three experiments in quantum optics.

The field operator transformations have been derived after a simple inspection of the graphs describing the experiments. The output state vectors have been obtained in a very fast and straightforward manner. Despite the simple model used, we obtained all the important features of these experiments, as described in the respective papers.

\section{A Density matrix approach}

Having the output state vector (7) allows one to compute the output density matrix operator

$$
\hat{\rho}_{\text {out }}=\left|\psi_{\text {out }}\right\rangle\left\langle\psi_{\text {out }}\right|
$$

Since in this experiment we only use two output ports $(s$ and $i$ ), we partially trace $\hat{\rho}_{\text {out }}$ over the two unused outputs $\left(s^{\prime}\right.$ and $\left.i^{\prime}\right)$ yielding

$$
\hat{\rho}_{s, i}=\operatorname{Tr}_{s^{\prime}, i^{\prime}}\left\{\hat{\rho}_{\text {out }}\right\}=\sum_{m, n=0}^{\infty}\left\langle m_{s^{\prime}} n_{i^{\prime}} \mid \psi_{\text {out }}\right\rangle\left\langle\psi_{\text {out }}\right| m_{s^{\prime}} n_{i}
$$

and after some straightforward calculations we arrive at the final expression

$$
\begin{aligned}
\hat{\rho}_{s, i}=\frac{1}{2}( & \sin ^{2}\left(\varphi^{\prime}\right)\left|1_{s} 1_{i}\right\rangle\left\langle 1_{s} 1_{i}\left|+\cos ^{2}\left(\varphi^{\prime}\right)\right| 1_{s} 0_{i}\right\rangle\left\langle 1_{s} 0_{i}\right| \\
& \left.+\cos ^{2}\left(\varphi^{\prime}\right)\left|0_{s} 1_{i}\right\rangle\left\langle 0_{s} 1_{i}\left|+\sin ^{2}\left(\varphi^{\prime}\right)\right| 0\right\rangle\langle 0|\right)
\end{aligned}
$$

\section{References}

1. C. K. Hong, Z. Y. Ou, L. Mandel, Phys. Rev. Lett., 59, 2044 (1987)

2. T. Pittman et al., Phys. Rev. Let. 77, 10 (1996)

3. Z. Y. Ou, X. Y. Zou, L. J. Wang, L. Mandel, Phys. Rev. Lett., 65, 321 (1990)

4. S. Walborn et. al., Phys. Rev. A 65, 033818 (2002)

5. P. Grangier, G. Roger, A. Aspect, Europhys. Lett., 1, 173 (1986)

6. A. C. Elitzur, L. Vaidman, Found. Phys. 23, 987 (1993)

7. P.G. Kwiat, et al., Phys. Rev. Lett., 74, 4763 (1995) 
8. D. C. Burnham, D. L. Weinberg, Phys. Rev. Lett. 25, 84 (1970)

9. D. N. Klyshko, Sov. Phys. JETP Lett. 6, 23 (1967)

10. C. K. Hong, L. Mandel, Phys. Rev. A, 31, 2409 (1985)

11. M. H. Rubin, D. N. Klyshko, Y. H. Shih, A. V. Sergienko, Phys. Rev. A, 50, 5122 (1994); W. P. Grice, I. A. Walmsley, Phys. Rev. A, 56, 1627 (1997); T. E. Keller, M. H. Rubin, Phys. Rev. A, 56, 1534 (1997)

12. P. Kwiat, K. Mattle, H. Weinfurter, A. Zeilinger, A. Sergienko, Y. Shih, Phys. Rev. Lett. 75, 4337 (1995)

13. Y. H. Shih, C. O. Alley, Phys. Rev. Lett. 61, 2921 (1988);

J. G. Rarity, P. R. Tapster, Phys. Rev. Lett. 64, 2495 (1990)

14. P. G. Kwiat, A. M. Steinberg, R. Y. Chiao, Phys. Rev. A, 47, R2472 (1993)

15. Z. Y. Ou, L. J. Wang, L. Mandel,Phys. Rev. A, 40, 1428 (1989)

16. Z. Y. Ou, L. J. Wang, X. Y. Zou, L. Mandel, Phys. Rev. A, 41, $566(1990)$

17. X. Y. Zou, L. J. Wang, L. Mandel, Phys. Rev. Lett. 67, $318(1991)$

18. L. J. Wang, X. Y. Zou, L. Mandel, Phys. Rev. A 44, 4614 (1991)

19. G. B. Lemos et al., Nature, 512, 409 (2014)

20. S. Ataman, Eur. Phys. J. D 68, 288 (2014)

21. R. Loudon, The Quantum Theory of Light, (Oxford University Press, Third Edition, 2003)

22. D. M. Greenberger, M. A. Horne, A. Zeilinger, Physics Today, p.26 (August 1993)

23. H. M. Wiseman, K. Mølmer, Physics Letters A 270, 245 (2000)

24. S. Ataman, Eur. Phys. J. D 68, 317 (2014) 